\section{Coagulación intravascular diseminada en loxoscelismo, realidad o mito}

\section{Disseminated intravascular coagulation in loxoscelism, reality or myth}

\section{Sr. Editor:}

Loxoscelismo se define como el conjunto de signos y síntomas o cuadro tóxico originado por el emponzoñamiento de un arácnido del género Loxosceles ${ }^{1}$. Entre los cuadros clínicos causados, destacan el loxoscelismo cutáneo y sistémico o visceral. El primero se caracteriza por la lesión cutánea-necrótica y el segundo, por el compromiso sistémico, basado en la hemólisis intravascular asociada a trombocitopenia con repercusión en diversos sistemas, como el renal ${ }^{2}$. La coagulación intravascular diseminada (CID) es un estado de activación y estimulación excesiva del sistema de coagulación, que origina una microangiopatía trombótica y fibrinólisis secundaria, como resultado patológico de alguna noxa. Es una de las manifestaciones y complicaciones del loxoscelismo, reportada en libros y textos científicos referidos al tema ${ }^{4,5}$. Pero, ¿qué tan confiable es este dato?. En realidad, no hemos evidenciado esta presentación en los cuadros vistos hasta hoy. El origen de este dato se basa en el reporte de un caso, publicado en el año 1972 por Vorse y cols. ${ }^{6}$, quienes describieron el cuadro en un niño tras una mordedura, mientras jugaba debajo de un muelle en un lago rural. Como característica principal, se observaron esquistocitos y esferocitos en el frotis sanguíneo. Un año después, en 1973, Berger y cols. ${ }^{7}$, publicaron un estudio experimental realizado en conejos, a quienes se les inoculó veneno a distintas dosis, mostrando como daño primario una vasculopatía a nivel del endotelio, previo al estado inflamatorio, con hemorragia y trombosis endotelial. En el conejo, la hemorragia y necrosis progresiva de la piel estuvieron asociadas a trombocitopenia, fibrinogenemia, y prolongación del tiempo de coagulación. Se concluyó que el origen de la alteración necrótica, hemorrágica y de coagulación se basaba en la toxicidad endotelial que surgía a las tres horas siguientes a la inoculación.

En Perú, en estudios de series de casos, como la de Maguiña, donde fueron incluidos un considerable número de pacientes, niños y adultos, no se encontró CID. La mortalidad se asoció a falla renal, por hemólisis intravascular grave, en pacientes con factores de riesgo (como edad menor de 1 año). En Brasil, en un estudio realizado por Malaque y cols. ${ }^{8}$, se analizaron 81 pacientes con diagnóstico de loxoscelismo cutáneo y sistémico, con pocos pacientes con manifestación visceral. En esta serie no se encontró CID, pero sí alteraciones como presencia de dímero $\mathrm{D}$ elevado y sólo en pocos, trombocitopenia.

En el laboratorio, la alteración plaquetaria en conejos está demostrada. In vivo existe trombocitopenia, sin embargo, in vitro no se muestra lisis ni agregación directa, sólo activación y posterior agregación por un factor sérico, refiriéndose a una función activadora plaquetaria9 ${ }^{9}$. McGlasson y cols. ${ }^{10}$ estudiaron a 36 conejos, divididos por grupos, a quienes se les inoculó veneno de Loxosceles. Se observaron alteraciones del sistema de coagulación, como disminución de la proteína $\mathrm{C}$ y elevación del TTPA, pero ningún cuadro de CID.

En resumen, sólo ha habido un reporte de CID hasta la fecha. No se ha evidenciado en estudios clínicos, sólo en casos excepcionales experimentales. En el caso reportado se sostiene una hipótesis de loxoscelismo, sin confirmación con un estudio entomológico, al igual que los estudios clínicos, evidenciando un sesgo no confiable. Si debe seguir siendo considerado esta asociación en los textos científicos, es una cuestión que debe ser aclarada en su citación. A futuro debería realizarse un gran estudio para determinar su presentación. Por el momento, el CID en loxoscelismo es más un mito que una realidad.

\section{Referencias bibliográficas}

1.- Schenone H. Cuadros tóxicos producidos por mordeduras de araña en Chile: latrodectismo y loxoscelismo. Rev Med Chile 2003; 131: 437-44.

2.- Maguiña-Vargas C, Hinojosa J C, Gutiérrez R, Henríquez C, Ugarte C. Enfermedades por artrópodos. Parte I: Loxoscelismo cutáneo y cutáneo-visceral en el Perú. Dermatol Peru 2004; 14: 134-9.

3.- Gremski L H, Trevisan-Silva D, Ferrer V P, Matsubara F H, Meissner G O, Wille A C, et al. Recent advances in the understanding of brown spider venoms: from the biology of spiders to the molecular mechanisms of toxins. Toxicon 2014; 83: 91-120.

4.- Páramo J A. Coagulación intravascular diseminada. Med Clin (Barc) 2006; 127: 785-9.

5.- Juckett G. Arthropod bites. Am Fam Physician 2013; 88: 841-7.

6.- Vorse H, Seccareccio P, Woodruff K, Humphrey G B. Disseminated intravascular coagulopathy following fatal brown spider bite (necrotic arachnidism). J Pediatr 1972; 80: 1035-7.

7.- Berger R S, Adelstein E H, Anderson P C. Intravascular coagulation: the cause of necrotic arachnidism. J Invest Dermatol 1973; 61: 142-50.

8.- Malaque C M, Santoro M L, Cardoso J L, Conde M R, Novaes C T, Risk J Y, et al. Clinical picture and laboratorial evaluation in human loxoscelism. Toxicon 2011; 58: 664-71.

9.- Tavares F L, Peichoto M E, Rangel D de M, Barbaro K C, Cirillo M C, Santoro M L, et al. Loxosceles gaucho spider venom and its sphingomyelinase fraction trigger the main functions of human and rabbit platelets. Hum Exp Toxicol 2011; 30: 1567-74.

10.- McGlasson D L, Harroff H H, Sutton J, Dick E, Elston D M. Cutaneous and systemic effects of varying doses of brown recluse spider venom in a rabbit model. Clin Lab Sci 2007; 20: 99-105.

Rafael Pichardo-Rodríguezy José Antonio Grandez-Urbina

Instituto de Investigación en Ciencias Biomédicas, Universidad Ricardo Palma. Lima, Perú.

Correspondencia a: Rafael Pichardo-Rodríguez rafael_martin1352@hotmail.com 